Article

\title{
Mechanical Damage Assessment for Pneumatic Control Valve based on Statistical Reliability Model
}

\author{
Nirbhay Mathur ${ }^{1} * \mathbb{D}$, Vijanth S. Asirvadam ${ }^{1}$ and Azrina Abd, Aziz ${ }^{1}$ \\ 1 Affiliation 1; Department of Electrical \& Electronics Engineering Univiersiti Teknologi PETRONAS \\ Seri Iskander, Malaysia. \\ * Correspondence: nirbhay.mathur@utp.edu.my
}

\begin{abstract}
Reliability assessment is an important component and tool used for process plants since the facility consists of many loops and instruments attached and operate based on each other availability, thus it requires a statistical method to visualize the reliability. The paper focuses on reliability assessment and prediction based on available statistical models such as normal, log-normal, exponential, and Weibull distribution. This paper also visualizes, which model fits best for assessment and prediction and also considers failure modes caused during a simulation mode process control operation. A simulation model is designed in this paper to observe the failure of the control valve causing stiction to visualize the failure modes and predict the best-fit model for reliability assessment.
\end{abstract}

Keywords: Fault detection; Control Valve; Reliability, Prediction.

\section{Introduction}

Process plants consist of a large number of machines to perform overall operations, and as the industries have already moved into the era of digitization, it is apprehended to automatize all process operations. In this challenging world, in order to keep operations in the reliable mode, it is required to look into all parameters which affect cost of overall operating mainly damage assessments. Reliability assessment is an important phase of any plant. Since each machine/plant consist of a large number of sub-systems and each sub-system have many large components attached with each other.

Reliability analysis can be performed by both qualitative and quantitative [1] measurements as reliability is a probability of equipment stayed operated without getting fail for a given time of interval [2] and the reliability of the plant/ machine mostly gets influenced by the method of operating which can be at the time of high production or scheduled outage [3].

Reliability study had been carried out by many researchers, and this study looks into control valve reliability in the process plant and research shows two major aspects of valve reliability such as failure analysis of the specific type of valves and methodology on fault and reliability [4]. Control valves are considered as the actuator for process regulation in plants. Since performance and reliability play a significant role to keep the process steady and accurate. It has been defined that about $30 \%$ of valve nonlinearities problems occur due to stiction, deadband, and hysteresis [5]. As the nonlinearity in the process plants increases the oscillation for the control valve to accelerate towards degradation which increase the operation cost and start producing lower output [6] and in way the long term can cause major losses to process production [7] [8].

This research paper is divided into four (4) major sections in which section one (1) addresses the introduction of reliability and control valve, whereas Section two (2) addresses the literature of control valve stiction and how it affects the performance of the plant. Section three (3) addresses the methodology used to develop stiction and statistical reliability prediction model, section four (4) finally concludes and discussion on future occurring of stiction. 


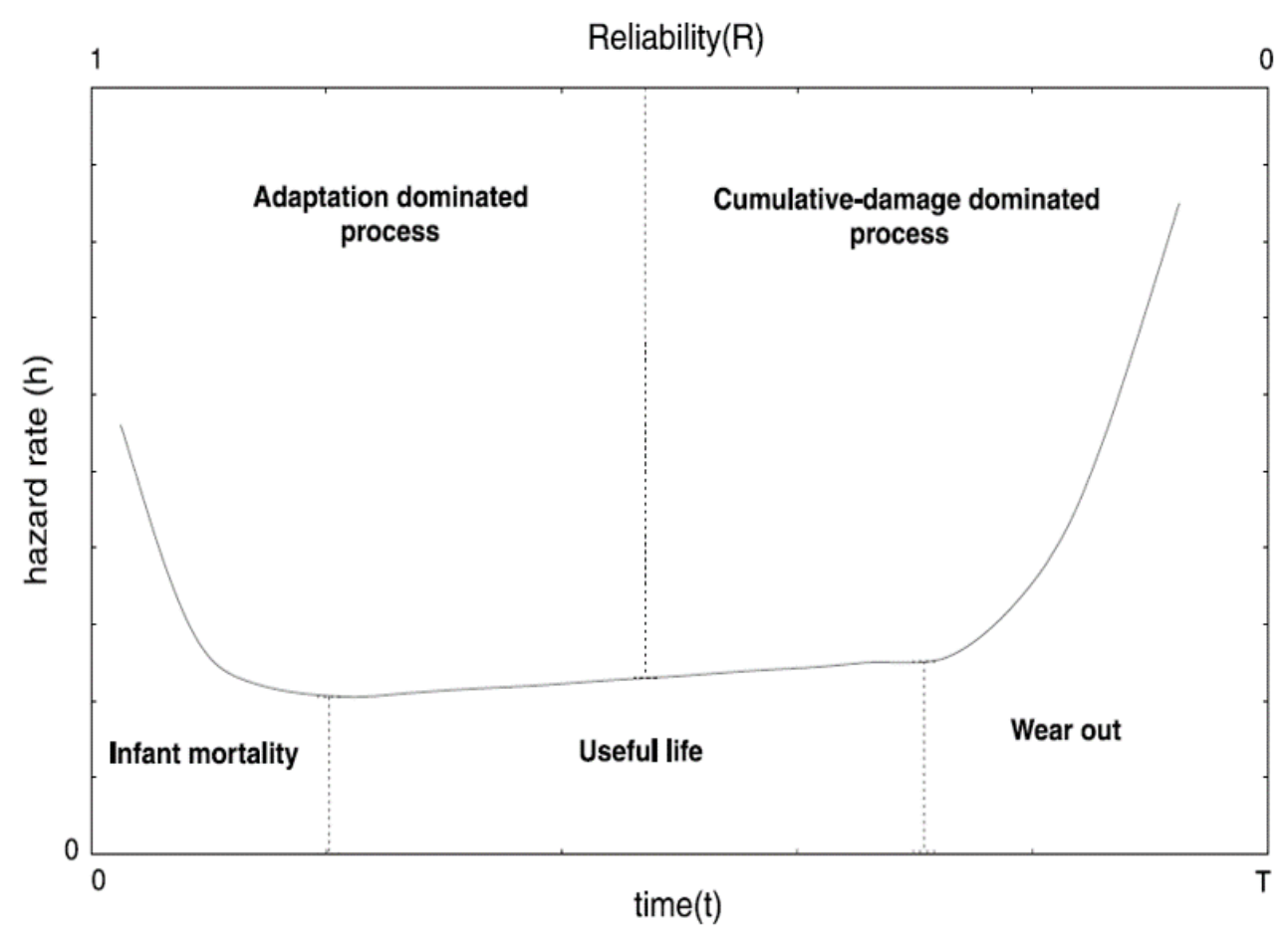

Figure 1. BathTub curve with three (3) phases.

\section{Reliability Assessment and its Components}

\subsection{Reliability Assessment}

Reliability assessment is a term that relates to evaluating the capability of a process in the industry to obtain good performance in mentioned service time.

To maintain a long life, low cost for maintenance and to achieve a high-profit rate it is required to design reliability assessment properly [9]. The other components of reliability assessment are availability and maintainability which together form RAM measurement.

\subsection{Availability}

A measure of percentage for machinery or equipment which are in operable and committable state for any time when the operation is required. The definition includes two major factors that are "operable" and "committable" which purely depends on the equipment itself. Availability is depended on the process and downtimes which are considered for analysis. Hence the classification of availability is stated as follows,

Inherent Availability, $A_{I}$ is a stage when correct downtime of system is planed. Inherent availability is denoted as $A_{I}$. It puerile depends on the design of equipment and this type of availability can be driven by the analysis of an design engineer. For single component in full process setup $A_{I}$, can be represented as:

$$
A_{I}=\frac{M T T F}{M T T F+M T T R}
$$

Achieved Availability, $A_{A}$ This availability is similar to inherent availability and function mostly same except the preventive maintenance (PM) downtime. PM is included in this availability. The achieved 
availability is something which is always seen by maintenance department to ensure the correctives of operation. Achieved availability can be formulated as follows:

$$
A_{A}=\frac{M T B M}{M T B M+\bar{M}}
$$

where,

$$
\begin{aligned}
& \quad M T B M=\text { Uptime } /(\text { NumberofSystemFailure }+ \text { NumberofSystemDowningPMs }) \\
& \bar{M}=(\text { CMDowntime }+ \text { PMDowntime }) /
\end{aligned}
$$

(NumberofSystemFailure + NumberofSystemDowningPMs)

Operational Availability, $A_{O}$ Operational Availability the real average over a period of time that includes all kind of downtime such as administrative, logistics downtime, etc.... It is an posterior availability based on actual events happening in system. All above discussed classification of availability can be assumed as prior estimated.

Mathematically this availability can be expressed as follows:

$$
A_{O}=\frac{\text { Uptime }}{\text { OperatingCycle }}
$$

Operational availability can be related to the concept of readiness operation. This type of classification is closely used in military application, where they assign the number of operating and maintenance personal to take care if any failure occurs.

\subsection{Maintainability}

A system is called maintained when the probability of that system is fully operational within a specific period of time $[15,16]$. Also, maintainability can be defined as a set of original activities that are carried out to keep the operation functionally with minimum cost acquired.

The procedure for the maintainability analysis of the system described in MIL-HDBK 472 [17] are described in following points:

- determining the relative frequency of failure modes

- obtaining estimates for elemental activities

- factoring by relative contribution

- summing factored time to give mean corrective/preventive time.

The MTBF and MTTR of the system can be calculated respectively as:

$$
M=\frac{M T T R}{M T B F+M T T R}
$$

where, M=Maintainability

MTTR= Mean Time To Repair,

$\mathrm{MTBF}=$ Mean Time Between Failure.

\subsection{Bath Tub Curve}

The graphical representation of failure rate over time is named as Bathtub curve. This names was given as the plot resembles like bathtub shape. This type of curve is used to represent the lifetime of product/ component and describes the failure pattern according the aging of product based on time. This curve is divided into three main cycle phases: (1) The infant mortality region, when the system is newly introduced and has a high failure rate; (2) The constant failure rate, when the product stable 


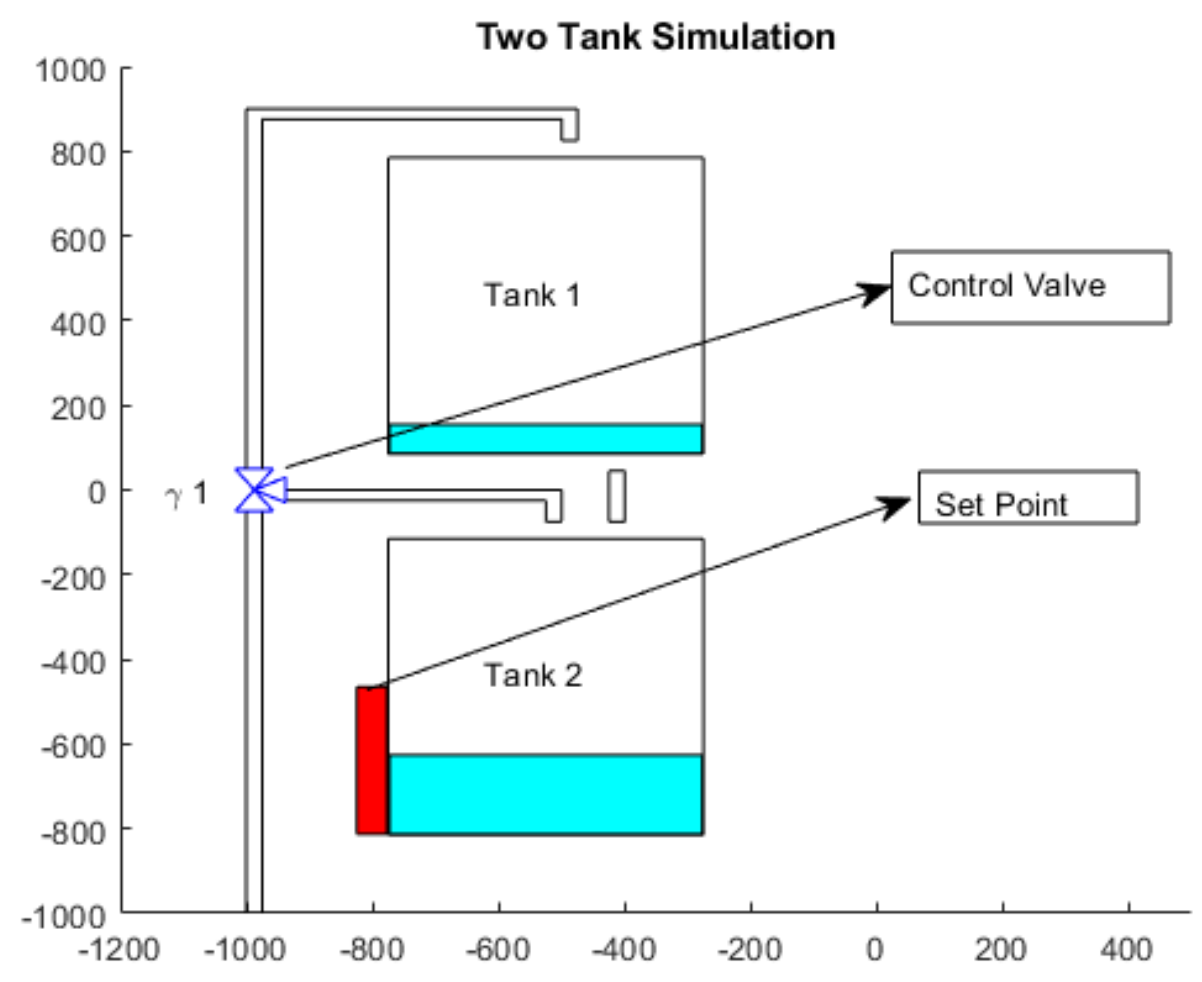

Figure 2. Two Tank system simulation

with low failures; (3) The wear-out region, when the failure rate is significantly increased. Figure.1 represents the three phases based on reliability vs time.

\section{Methodology \& Results}

This section will discuss about the methodology used in predicting the reliability for control valve and visualize using different statistical models.

\subsection{Experiment Setup-Two Tank Control Valve}

In this research, two tank system is considered as the experimental set up. Along with two tank an control valve is place to control the flow of rate at different gate valve opening levels. This setup was designed in MATLAB environment using SIMULINK, as shown in Figure 2. As per the survey, 30\% of failure occurs in process plants are due to control valves. These failures happen due to the nonlinearity of systems such as stiction or deadband $[9,10]$. Since the percentage of failure is considered bigger hence this failure can decrease productivity and lower the profitability $[10,11]$. Valve stiction problem is easily confused with other nonlinearity problems such as deadband, backlash, hysteresis as shown in Figure $3[12,13]$.

\subsection{Data Collection}

In order to conduct the experiment, the failure data was collected from two tank system in which stiction was originated at some random points. The data was divided in to three (3) section in which the first (1) section define numbers of hours control valve operated. The second (2) section recorded event, which define about failure and the third (3) and last section was count in which rate of flow was defined by using set point in simulation environment. These data represent the failure of a control valve due to stiction produced while operating. 


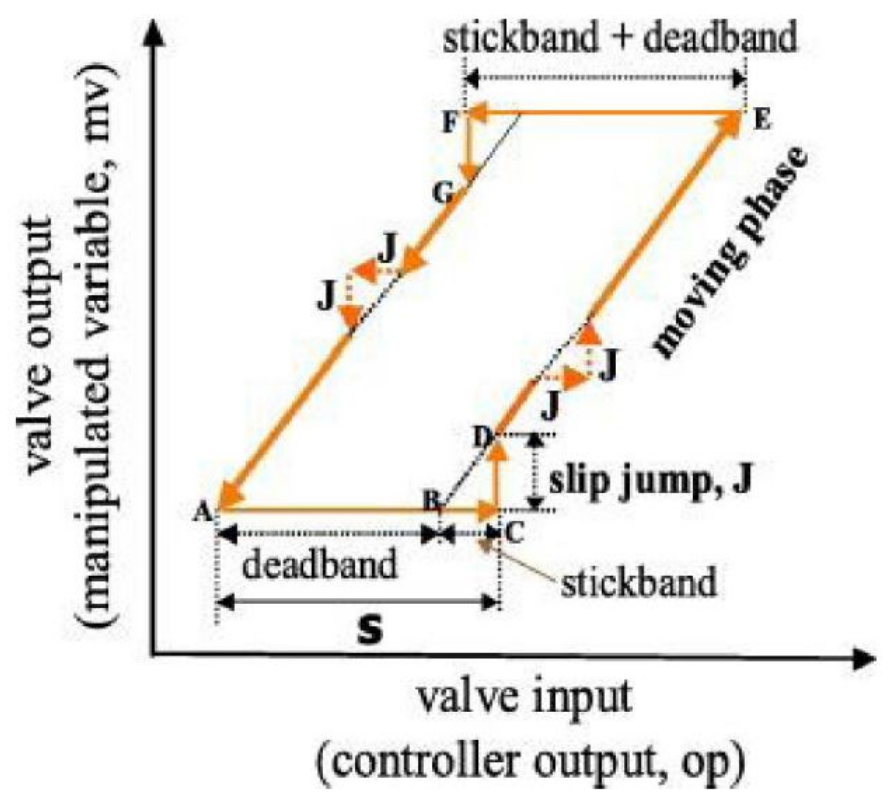

Figure 3. Valve stiction behavior.

The experiment was conducted on two type of data sets one which was simulated data on Matlab environment and another data collected from pilot plant. As shown in Figure 4 and Figure 5 shows the flow chart for data storage from pilot plant. Figure 6 shows the output of the pilot plant and sudden occurrence of stiction, which make overshoot in output. After understanding the nature of failure mode in data set, it is required to further process with prediction modeling.

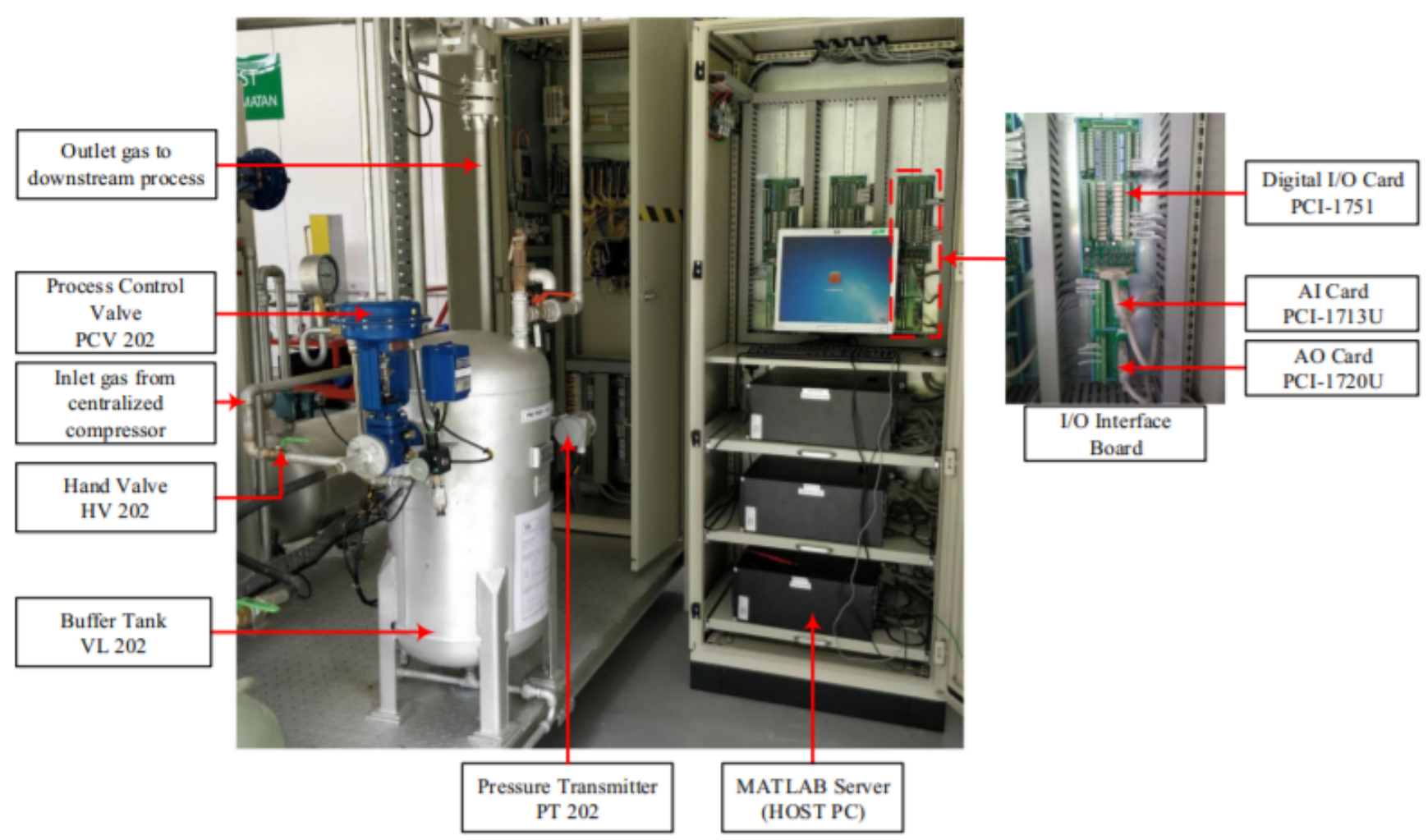

Figure 4. Pilot plant setup for data collection 


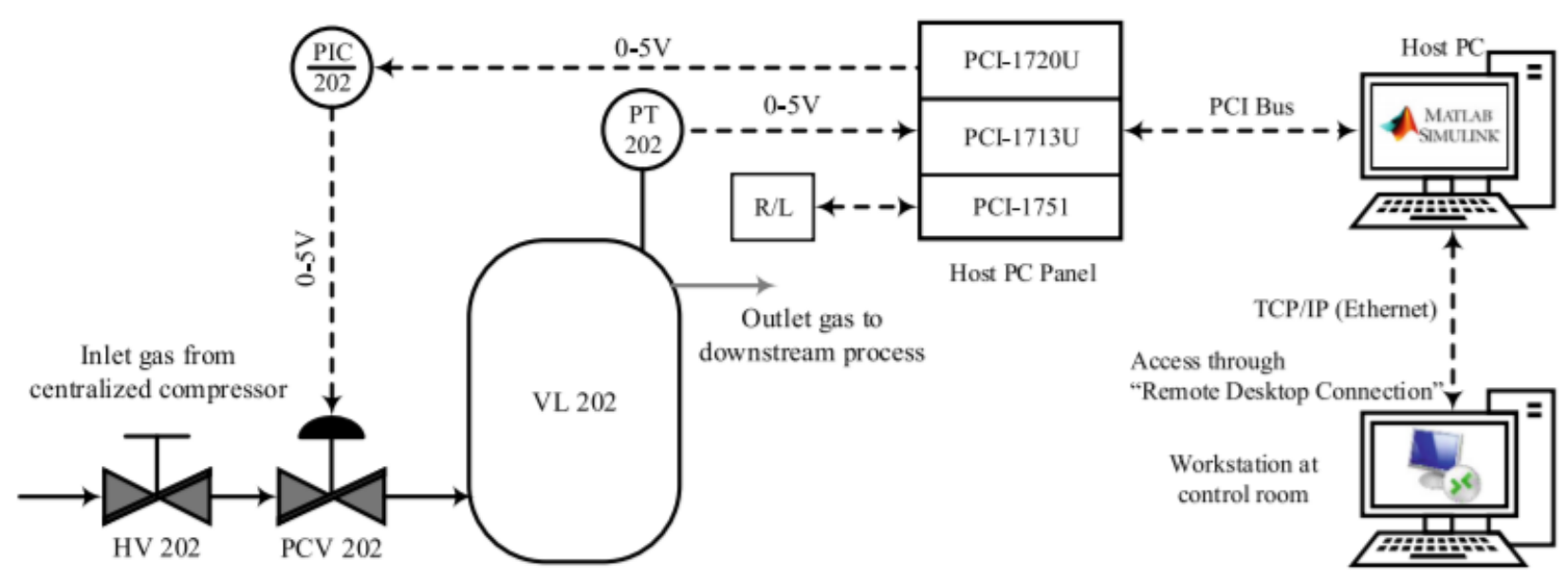

Figure 5. Data extraction flowchart.

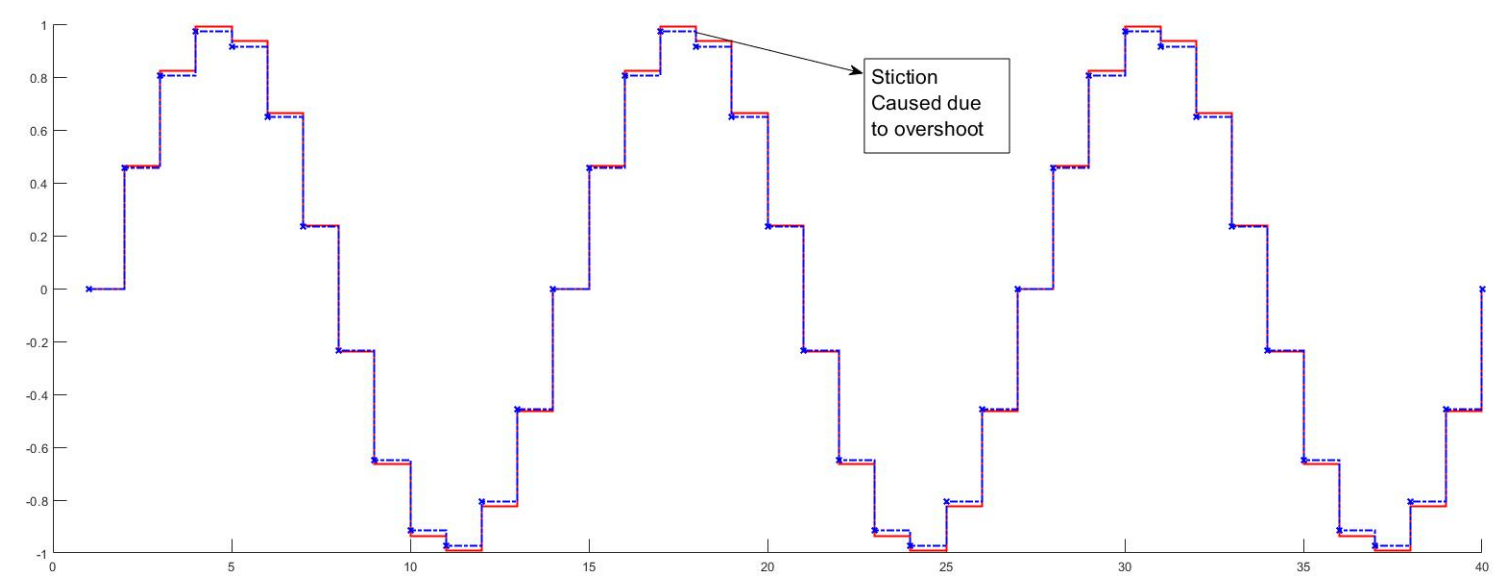

Figure 6. Control valve operation plot to see behavior of stiction in comparison of set point.

\subsection{Reliability Prediction}

To visualize the reliability of control valve data, TTF (Time to Failure) measurement was predicted using the Bayesian distribution and Weibull distribution. The assumptions for choosing reliability models(such as; Weibull, lognormal, exponential, etc..) depend on different cases and different type of data sets.

\subsubsection{Weibull Distribution}

The Weibull distribution cumulative density function (cdf) is written as

$$
\operatorname{Pr}(T \leq t ; \eta, \beta)=1-\exp \left[-\left(\frac{t}{\eta}^{\beta}\right)\right], t>0
$$

where, $\beta>0$ is a shape parameters and $\eta>0$ is a scale parameter. In particular, if $T$ has a Weibull distribution, then $Y=\log (T) \sim \operatorname{SEV}(\mu, \sigma)$, where $S E V$ is survival value, $\sigma=1 / \beta$ is the scale parameter and $\mu=\log (\eta)$ is the location parameter. Lets say, $T$ has a Weibull distribution, which can be represented by $T \sim \operatorname{WEIB}(\mu, \sigma)[14]$. Now the Weibull cdf, pdf and hf can be written as:

$$
F(t: \mu, \sigma)=\Phi_{s e v}\left[\frac{\log (t)-\mu}{\sigma}\right]
$$




$$
\begin{aligned}
& f(t ; \mu, \sigma)=\frac{1}{\sigma t} \Phi_{\text {sev }}\left[\frac{\log (t)-\mu}{\sigma}\right]=\frac{\beta}{\eta}\left(\frac{t}{\eta}\right)^{\beta-1} \exp \left[-\left(\frac{t}{\eta}\right)^{\beta}\right] \\
& h(t ; \mu \sigma)=\frac{1}{\sigma \exp (\mu)}\left[\frac{t}{\exp (\mu)}\right]^{\frac{1}{\sigma-1}}=\frac{\beta}{\eta}=\frac{\beta}{\eta}\left(\frac{t}{\eta}\right)^{\beta-1}, t>0 .
\end{aligned}
$$

\subsection{Normal Distribution}

Normal distribution can be derived, when $Y$ indicates $Y \sim N O R(\mu, \sigma)$. Pdf and cdf can be write for normal distribution is as follows:

$$
\begin{gathered}
F(y ; \mu, \sigma)=\Phi_{\text {nor }}\left(\frac{y-\mu}{\sigma}\right) \\
f(y ; \mu, \sigma)=\frac{1}{\sigma} \Phi_{\text {nor }}\left(\frac{y-\mu}{\sigma}\right),-\infty<y<\infty,
\end{gathered}
$$

where $\Phi_{\text {nor }}=(1 \sqrt{2 \pi}) \exp \left(-z^{2} / 2\right)$ and $\Phi_{\text {nor }}(z)=\int_{-\infty}^{z} \Phi_{\text {nor }}(w) d w$, also here $-\infty<y<\infty$ is a location parameter and $\sigma>0$ is the scale parameter.

Also, the normal distribution is well known for its simplicity and center limit application [14]. But when it comes to reliability analysis normal distribution is less used because of if hf rate calculation is valid till the median of the data. The normal distribution is useful only with some limited data set with limited life cycle data in which the following condition is true (i.e, $\mu>0$ ) and coefficient variation $(\sigma / \mu)$. A plot can be visualized in Figure 7 (a) for this research case study.

\subsection{Exponential Distribution}

Considering the same $T$ from equation 1, exponential distribution can be denoted as $T \sim$ $\operatorname{EXP}(\theta, \gamma)$. The two parameter exponential distribution can be driven for cdf, pdf and hf function as follows:

$$
F(t ; \theta, \gamma)=1-\exp \left(-\frac{t-\gamma}{\theta}\right)
$$

equation 7 defines the cdf for the exponential distribution. Now by considering equation 7 we can derive pdf and hf function as follows:

$$
\begin{gathered}
f(t ; \theta, \gamma)=\frac{1}{\theta} \exp \left(-\frac{t-\gamma}{\theta}\right) \\
h(t ; \theta, \gamma)=\frac{1}{\theta}, t>\gamma
\end{gathered}
$$

ere, $\theta>0$ is considered as a scale parameter and $\gamma$ is a location parameter. As per [14] exponential distribution is most commonly used in electronics components. Whereas this distribution is not suitable if the data set has component failure data due to defect. In our case study where control valve has stiction, which is a cause of failure that can be parked in mechanical failure. The exponential distribution plot can be visualized in Figure $7 \mathrm{c}$.

\subsection{Lognormal Distribution}

Last comparison was made with lognormal distribution, this can be denoted as by considering the same assumption from equation $1, T \sim \operatorname{LOGNOR}(\mu, \sigma)$. If $T \sim \operatorname{LOGNOR}(\mu, \sigma)$ then $Y=\log (T \sim$ $\operatorname{NOR}(\mu, \sigma)$. The cdf and pdf for lognormal distribution can driven as follows:

$$
F(t ; \mu, \sigma)=\Phi_{\text {nor }}\left[\frac{\log (t)-\mu}{\sigma}\right]
$$

and

$$
f(t ; \mu, \sigma)=\frac{1}{\sigma t} \Phi_{\text {nor }}\left[\frac{\log (t)-\mu}{\sigma}\right], t>0
$$




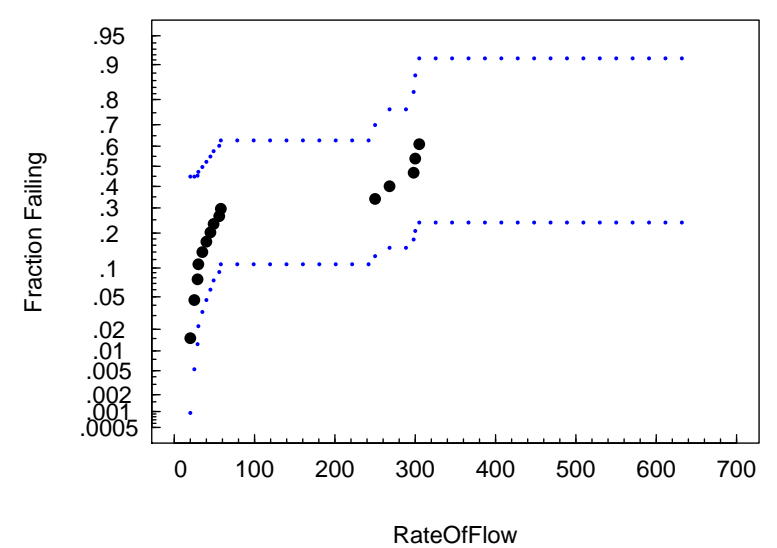

(a) Reliability plot for Normal distribution.

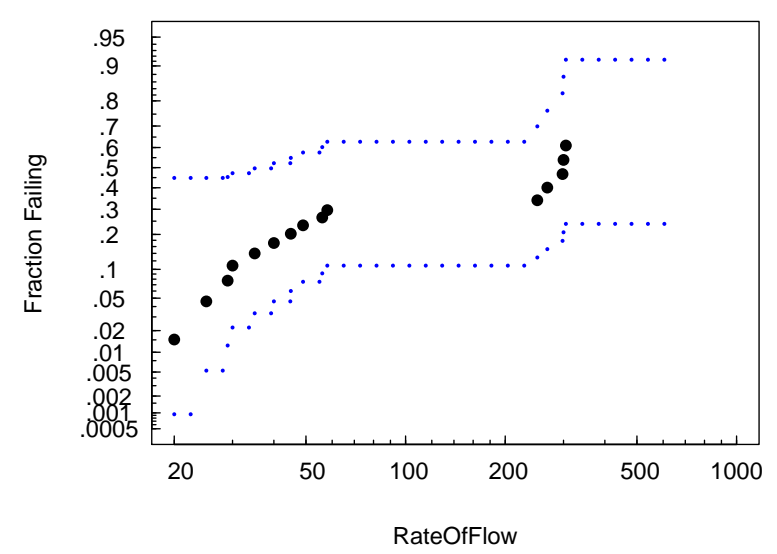

(c) Reliability plot for Exponential distribution.

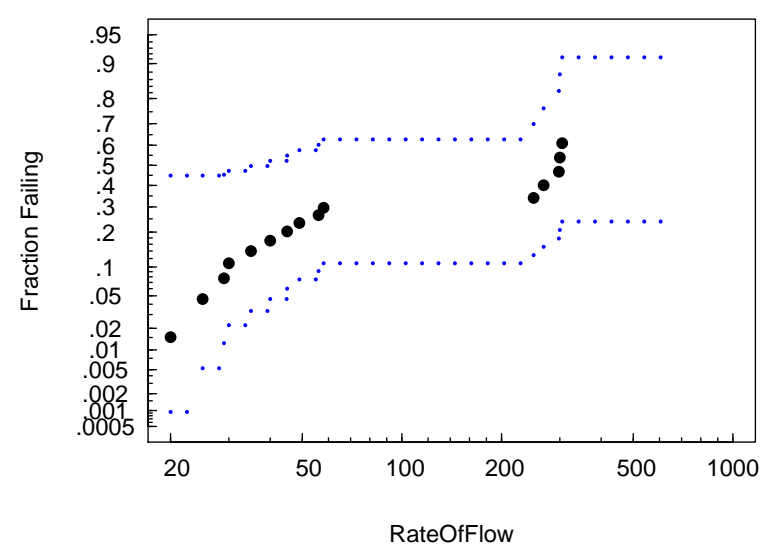

(b) Reliability plot for Lognormal distribution.

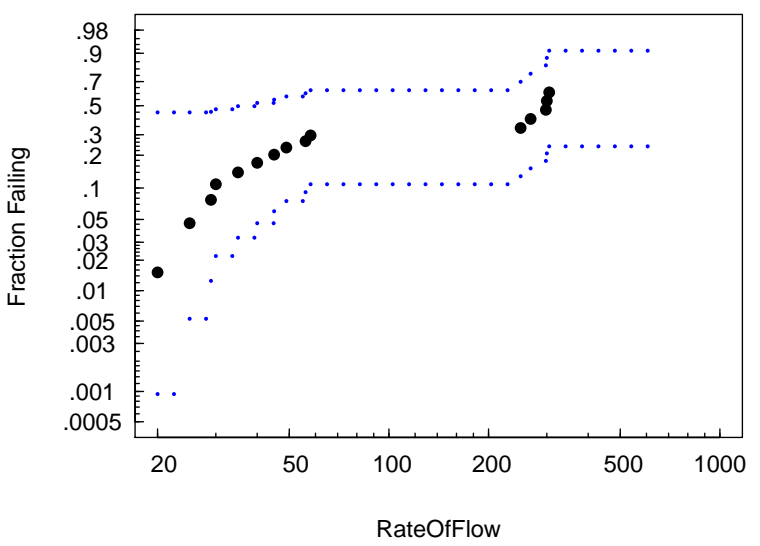

(d) Reliability plot for Weibull distribution.

Figure 7. Plot to visualize the different distribution fit with failure data.

where, $\Phi_{\text {nor }}$ and $\Phi_{\text {nor }}$ are pdf and cdf for standard normal distribution. This distribution is commonly used for failure times data set. It is suggested by system engineer and reliability engineers to consider lognormal distribution as it a model appropriate for degradation process failure.

Table 1. Failure rate calculated based on different stages of control valve gate opening for pilot plot data.

\begin{tabular}{ccccc}
\hline Rate of Flow & Fhat & Std.Error & \multicolumn{2}{c}{ MLE 95\% CI } \\
\hline & & & $95 \%$ Lower & $95 \%$ Upper \\
20 & 0.04945167 & 0.03025560 & 0.01471353 & 0.1593042 \\
50 & 0.10078031 & 0.04481346 & 0.04147054 & 0.2338855 \\
100 & 0.16959199 & 0.05828572 & 0.08482773 & 0.3226762 \\
200 & 0.27755249 & 0.07851267 & 0.08482773 & 0.3226762 \\
500 & 0.49386968 & 0.13000931 & 0.27755502 & 0.7598024 \\
1000 & 0.69617176 & 0.16954764 & 0.37852058 & 0.9493920 \\
\hline
\end{tabular}


Table 2. A comparison table between the prediction models for reliability and their calculated values.

\begin{tabular}{ccccc}
\hline Model & MTTF & \multicolumn{2}{c}{ Model 95\% CI } & Likelihood Value \\
\hline & & $95 \%$ Lower & $95 \%$ Upper & \\
Weibull Distribution & 652.6 hours & 106.5 & 4000 & -42.74 \\
Normal & 253.1 hours & 132.3 & 373.9 & -42.69 \\
Lognormal & 8952 hours & 115.5 & 694103 & -42.95 \\
Exponential & 25450 hours & 9552 & 67809 & -44.58 \\
\hline
\end{tabular}

\subsection{Maximum Likelihood Estimation (MLE) of System Reliability}

After selecting the best fitting model for our data set, it is required to perform the classification for the model and MLE is used in this paper to validate the various reliability model.

Lets us say,sample data are available to estimate the failure-time distributions of the system's individual components. For example, data on component $\mathrm{i}$ for $\mathrm{i}=l, \ldots, . s$ can be used to estimate $\theta_{t}, i=1, \ldots, s$, providing estimates $\hat{F}_{1}, \ldots, \hat{F}_{t}$, respectively. The cdf can be driven from [14]. The system $\operatorname{cdf}$ (or other related functions) can be estimated as functions of $\hat{F}_{1}, \ldots, \hat{F}_{t}$, the function being determined from the system's structure $[14,16]$. Let $\hat{\theta}$ be the ML estimate of $\theta$ (the unique parameters describing the components' cdfs) and $\hat{\Sigma}_{\hat{\theta}}$ the ML estimate of $\sum_{\hat{\theta}}$ obtained from the component data. Since, this process will provide the validation of 2-parameter Weibull distribution [17,18].

Process data with failure elements were modeled to fit all the above-mentioned models i.e., Normal distribution, Exponential distribution, Lognormal distribution, and Weibull distribution. Figure 7(a) shows the predicted reliability fit the Normal distribution by populating the failure points near to the lower and upper limit of prediction, which means the plot shows failure point but was not able to distinguish between individual parameters to predict reliability. Figure 7(b) shows the lognormal distribution, linearity parameters fit but the points are not distributed. Figure 7(c) \& Figure 7 (d) shows the Exponential and Weibull distribution in which both plots look quite similar. The Weibull plot provides detailed information about data fitting with a large amount of uncertainty expressed by confidence bands.

After plotting, confidence intervals values were calculated which are based on different input from gate valve opening levels. Table 1 shows $95 \%$ confidence intervals calculated from MLE for different inputs. In Table 1 standard error is calculated to keep track of confidence intervals prediction. Followed by Table 2 shows the computed predicted reliability values for different distributions such as Weibull, Normal, Lognormal, Exponential. Table 2 also computes the value for Mean Time to Fail which helps to provide an alert/alarm for which system can fail. Also, 95\% confidence intervals are calculated for a lower and upper band in which the system will be having a range to predict the failure. The range shown in Table 2 is based on reliability models, which shows the occurrence of failure based on time (hours). Table 3 shows the computational calculation of reliability prediction parameters such as shape and size parameters i.e, $\mu$ and $\sigma$ respectively. These values are calculated based on MLE and the $95 \%$ confidence intervals based of each model.

To understand the behavior and working of Weibull distribution, the calculated values for $\eta$ and $\beta$ were used to plot the hazard $h(t)$ function. As shown in Figure 8, both survival s(t) and hazard $h(t)$ shows the perfect fit for the bathtub curve. As discussed in Figure 1 the curve shows stage I and III. Since the survival plot shows the best working stage for the equipment and as the age of equipment starts to drain it will show the hazard curve with a random spike in failure.

\section{Conclusion \& Discussion}

The main aim of this research paper is to detect and visualize the fault and do reliability assessment for pneumatic control valve which is used more often in an industrial process plant using various statistical models. The paper visualizes the best fit of the model for analyzing reliability prediction [19]. 
Table 3. Computed values based on different models and their parameters to predict reliability

\begin{tabular}{ccccc}
\hline Model parameters & MLE & Stand Err & \multicolumn{2}{c}{ Model 95\% CI } \\
\hline Weibull $\mu$ & 6.6012 & 0.9328 & 4.773 & 8.4295 \\
Weibull $\sigma$ & 0.4108 & 0.1584 & 0.193 & 0.8745 \\
Weibull (eta $\eta$ ) & 736.0062 & 686.5469 & 118.272 & 4580.1809 \\
Weibull (beta $\beta$ ) & 2.4341 & 0.9383 & 1.143 & 5.1814 \\
Normal $\mu$ & 253.12 & 61.64 & 132.30 & 373.9 \\
Normal $\sigma$ & 66.14 & 21.80 & 34.66 & 126.2 \\
& & & & \\
Lognormal $\mu$ & 8.097 & 1.4848 & 5.1867 & 11.007 \\
Lognormal $\sigma$ & 1.416 & 0.5233 & 0.6864 & 2.922 \\
& & & & \\
Exponential $\mu$ & 10.14 & 0.5 & 9.164 & 11.12 \\
Exponential $\sigma$ & 1.00 & 0.0 & 1.000 & 1.00 \\
\hline
\end{tabular}
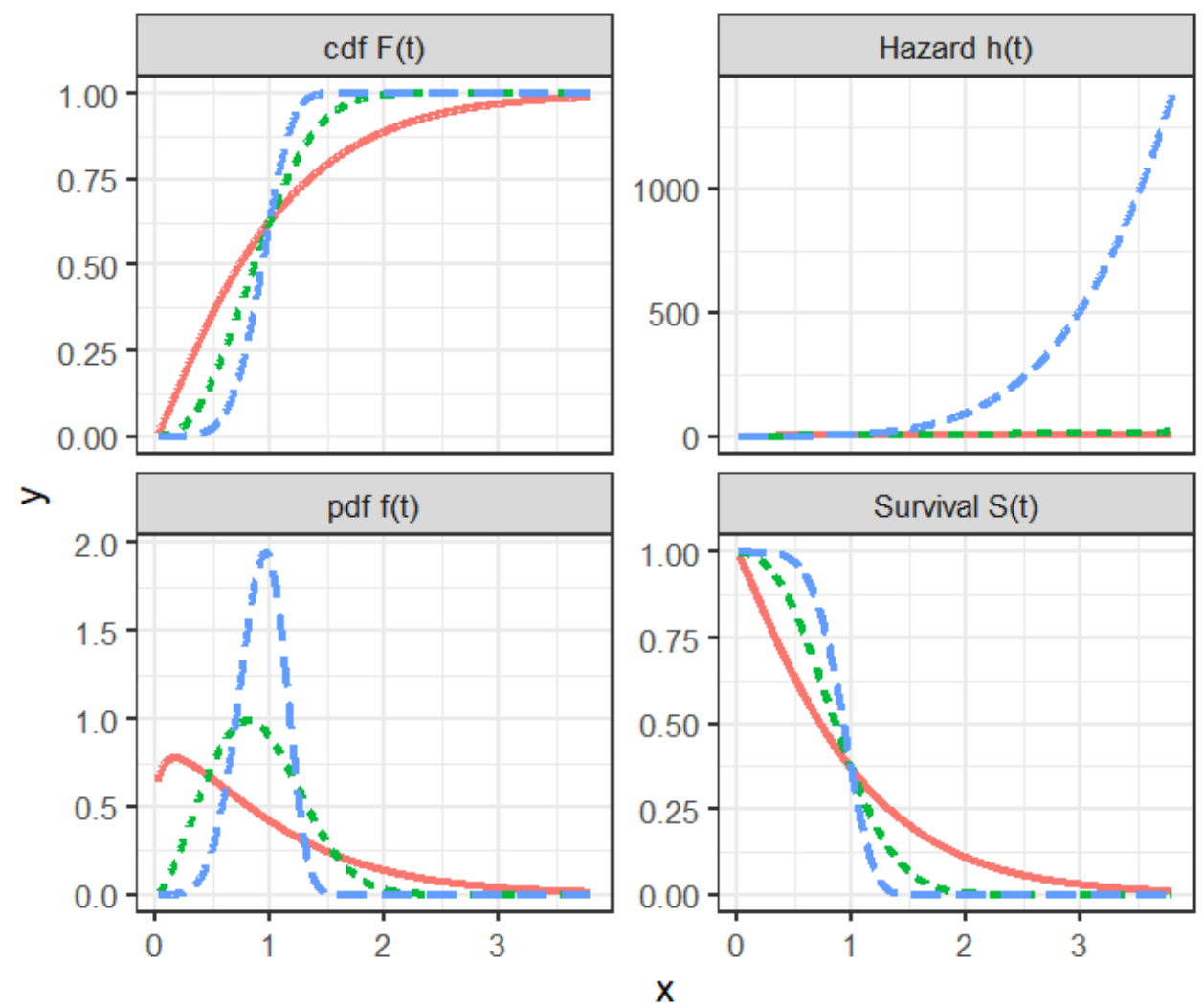

parameters

$-\beta, \eta=1.1430,1.00$

- $\beta, \eta=2.4341,1.00$

$-\beta, \eta=5.1810,1.00$

Figure 8. Weibull distribution for cdf, hazard, pdf and survival fit

The work also focuses on how to visualize the different types of failure modes that can affect together and also how the failure modes are correlated to each other. The data set was simulated with two-tank systems in which a pneumatic control valve was simulated. Along with that this paper also simulated the very common cause of failure for the control valve known as stiction [20]. The simulation has complied with different levels of input based on different gate valve opening and data was collected for the failure. After data collection, it is important to fit the data to best predict reliability. This paper discusses four major distributions and how they are liked with the prediction of reliability. Normal, Log-normal, Exponential, and Weibull distributions were modeled computationally to achieve the prediction score. Moreover referring to results from the above-mentioned table and plots, MTTF 
was predicted for the upper and lower band of operating hours. From all four(4) mentioned models, Weibull MTTF was considered as reliable predict as other models either predict for long time intervals or short time intervals. Weibull was able to give $95 \%$ confidence level prediction with an upper and lower limit, which was very similar to the data set. Also, results were validated by using the MLE algorithm for all four (4) models, which shows the best-predicted values for Weibull. Weibull has shape and size parameters $\eta$ and $\beta$ respectively, which help to understand the bathtub curve. The computed values for $\eta$ and $\beta$ were plotted for hazard $h(t)$ and survivals $(t)$ function. Hence, the conclusion was if the control valve work continuously with a change of gate valve opening levels, there is a change of originating stiction which can occur at time 652.6 hours of operation. To prevent the major shutdown first alarm will be notified at the time 106.5 hours, so that system could get an alert for occurring failure, also if the first warning is missed, the second alert will be generated at 4000 hours of operation to perform maintenance. Hence this will help to system engineers to prevent from sudden shutdown or closure of process plant.

Hence, this research is a complete package for reliability prediction including prediction and visualization which will help the system engineer to understand the reliability assessment and when it is required to perform the maintenance to keep the process plant in working condition. It will also help the process plant to maintain its production and cost-efficiency. Since this research is at a new stage of development, the author also suggests developing a smart dashboard that could be used for any type of instrument or component to understanding the working behavior and their reliability.

Author Contributions: "Conceptualization, N.M; methodology, N.M; software, N.M and V.S.A; validation, N.M; formal analysis, N.M and V.S.A; investigation, N.M; resources, V.S.A, A.A.A and R.b.I; data curation, N.M; writing-original draft preparation, N.M; writing-review and editing, V.S.A and A.A.A; visualization, N.M; supervision, V.S.A; project administration, V.S.A and N.M; funding acquisition, V.S.A and N.M. All authors have read and agreed to the published version of the manuscript.".

Funding: "This research was funded by Yayasan Universiti Teknologi PETRONAS grant number YUTP(015LC0-125).".

Acknowledgments: First author wants thanks Universiti Teknologi PETRONAS for providing resources. Author also want to show gratitude to all co-authors for providing their support and help in conducting this research. All codes and plots are generated using $R$ statistical programming packages.

Conflicts of Interest: Declare conflicts of interest or state "The authors declare no conflict of interest."

$\begin{array}{ll}\text { Abbreviations } \\ \text { The following abbreviations are used in this } \\ \text { IR } 4.0 \quad \text { Industry Revolution } 4.0 \\ \text { MTTF } & \text { Mean Time To Fail } \\ \text { MTTR } & \text { Mean Time To Repair } \\ \text { MTBF } & \text { Mean Time Between Failure } \\ \text { TTF } & \text { Total Time to Fail } \\ \eta & \text { eta } \\ \beta & \text { beta } \\ \text { SEV } & \text { Survival } \\ \sigma & \text { sigma } \\ \mu & \text { mu } \\ \text { WEIB } & \text { Weibull Distribution } \\ \text { CDF } & \text { Cumulative Density Function } \\ \text { PDF } & \text { Probability Density Function } \\ \text { Hf } & \text { Hazard Function } \\ \text { MLE } & \text { Maximum Likelihood Estimation }\end{array}$




\section{References}

1. Morcos, Maged. "Assessing systems reliability: a Probabilistic Risk Assessment (PRA) approach." In IN: Proceedings of 18th AR2TS, Advances in Risk and Reliability Technology Symposium Loughborough University, pp. 21-23. 2009.

2. Kumar, Dhananjay, Bengt Klefsjo, and Uday Kumar. "Reliability analysis of power transmission cables of electric mine loaders using the proportional hazards model." Reliability Engineering \& System Safety 37, no. 3 (1992): 217-222.

3. Warburton, D., J. E. Strutt, and K. Allsopp. "Reliability prediction procedures for mechanical components at the design stage." Proceedings of the Institution of Mechanical Engineers, Part E: Journal of Process Mechanical Engineering 212, no. 4 (1998): 213-224.

4. Han, Fei, Bifeng Song, Tianxiang YU, and Weimin CUI. "Present State and Prospects for Valve Reliability Technique Study [J]." Machine Tool \& Hydraulics 9 (2008): 48.

5. Desborough, Lane, and Randy Miller. "Increasing customer value of industrial control performance monitoring-Honeywell's experience." In AIChE symposium series, no. 326, pp. 169-189. New York; American Institute of Chemical Engineers; 1998, 2002.

6. Shinskey, F. Greg. "Putting controllers to the test." Chemical engineering 97, no. 12 (1990): 96.

7. Martin, G. D., L. E. Turpin, and R. P. Cline. "Estimating control function benefits." Hydrocarbon processing (International ed.) 70, no. 6 (1991): 68-73.

8. Miao, Tina, and Dale E. Seborg. "Automatic detection of excessively oscillatory feedback control loops." In Proceedings of the 1999 IEEE International Conference on Control Applications (Cat. No. 99CH36328), vol. 1, pp. 359-364. IEEE, 1999.

9. Yang, Jian-Wei, Jin-Hai Wang, Qiang Huang, and Ming Zhou. "Reliability assessment for the solenoid valve of a high-speed train braking system under small sample size." Chinese Journal of Mechanical Engineering 31, no. 1 (2018): 47.

10. Martin, G. D., L. E. Turpin, and R. P. Cline. "Estimating control function benefits." Hydrocarbon processing 70, no. 6 (1991): 68-73.

11. Miao, Tina, and Dale E. Seborg. "Automatic detection of excessively oscillatory feedback control loops." In Control Applications, 1999. Proceedings of the 1999 IEEE International Conference on, vol. 1, pp. 359-364. IEEE, 1999.

12. Choudhury, MAA Shoukat, Nina F. Thornhill, and Sirish L. Shah. "Modelling valve stiction." Control engineering practice 13 , no. 5 (2005): 641-658.

13. Choudhury, MAA Shoukat, Mridul Jain, and Sirish L. Shah. "Stiction-definition, modelling, detection and quantification." Journal of Process control 18, no. 3-4 (2008): 232-243.

14. Meeker, William Q., and Luis A. Escobar. Statistical methods for reliability data. John Wiley \& Sons, 2014.

15. Andrews, John D., and Thomas Robert Moss. Reliability and risk assessment. Wiley-Blackwell, 2002.

16. Mathur, Nirbhay, Vijanth S. Asirvadam, Azrina Abd Aziz, and Rosdiazli Ibrahim. "Visualizing and predicting reliability of control valves based on simulation." In 2018 IEEE Conference on Systems, Process and Control (ICSPC), pp. 54-59. IEEE, 2018.

17. Andrews, John D and Moss, Thomas Robert"Reliability and Risk Assessment", Wiley-Blackwell, 2002.

18. Davis, Gordon B "Management Information Systems (MIS)" John Wiley and Sons Ltd, 2003.

19. Mathur, Nirbhay, Vijanth S. Asirvadam, Azrina Abd Aziz, and Rosdiazli Ibrahim. "Fault tree analysis for control valves in process plants by using R." In 2019 IEEE 15th International Colloquium on Signal Processing \& Its Applications (CSPA), pp. 152-156. IEEE, 2019.

20. Choudhury, M. A. A. S., Sirish L. Shah, and Nina F. Thornhill. "Detection and quantification of control valve stiction." IFAC Proceedings Volumes 37, no. 9 (2004): 865-870. 